\title{
Experiments on transverse compression of a long charge bunch in a linear Paul trap
}

\author{
Moses Chung, Erik P. Gilson, Mikhail Dorf, Ronald C. Davidson, Philip C. Efthimion, and Richard Majeski \\ Plasma Physics Laboratory, Princeton University, Princeton, New Jersey 08543, USA
}

(Received 20 March 2007; published 19 June 2007)

\begin{abstract}
The transverse compression of a long charge bunch is investigated in the Paul trap simulator experiment (PTSX), which is a linear Paul trap that simulates the nonlinear transverse dynamics of an intense charged particle beam propagating through an equivalent kilometers-long magnetic alternating-gradient (AG) focusing system. Changing the voltage amplitude at fixed focusing frequency in the PTSX device corresponds to changing the field gradient of the quadrupole magnets with fixed axial periodicity in the AG transport system. In this work, we present experimental results on transverse compression of the charge bunch in which the amplitude of the applied oscillatory focusing voltage is changed instantaneously, and adiabatically. The experimental data are also compared with analytical estimates and 2D WARP particle-in-cell simulations.
\end{abstract}

DOI: 10.1103/PhysRevSTAB.10.064202

PACS numbers: 41.85.Ja, 29.27.-a, 52.27.Jt, 29.27.Bd

\section{INTRODUCTION}

Applications of present- and next-generation highintensity accelerators [1-4] to high energy and nuclear physics, high energy density physics, and heavy ion fusion often require transverse and longitudinal compression of the charge bunch to a small spot size at the target location $[5,6]$. Typically, the transverse compression can be achieved by means of increasing the focusing strength of the alternating-gradient (AG) lattice along the beam propagation direction [5]. However, intense beam propagation through such a lattice transition region inevitably leads to a certain amount of beam mismatch and emittance growth $[2,3]$. Furthermore, a beam mismatch can produce halo particles and may deteriorate the beam quality $[7,8]$. Hence, it is of considerable practical importance to determine how smooth the lattice transition should be in order that the beam mismatch and emittance growth are minimized during the transverse compression process. Because high-intensity accelerator systems are typically very long and expensive to operate, dedicated experimental studies of transverse compression are limited in number and scope [9]. On the other hand, the Paul trap simulator experiment (PTSX), which is a linear Paul trap [10] that can simulate the nonlinear transverse dynamics of intense beam propagation over large equivalent distances through an AG transport lattice [11], provides a compact and flexible laboratory setup for the experimental investigation of transverse compression. The amplitude and frequency of the voltage waveform applied to the electrodes of the PTSX device correspond to the focusing strength and lattice spacing in an AG system, respectively. The computer-generated arbitrary waveform of the voltage amplitude can emulate various types of transition patterns. Hence, in this study, we present experimental results describing the transverse compression of an intense beam pulse by compressing a long nonneutral ion charge bunch trapped in the PTSX device. In addition to compression, the results of expansion ("decompression") experiments are also presented, and comparisons are made among analytical theory, experimental results, and numerical simulations.

\section{PAUL TRAP SIMULATOR EXPERIMENT (PTSX)}

The PTSX device, cesium ion source, and diagnostics have been described in detail elsewhere $[12,13]$ and only a brief summary is given here. To generate the oscillating quadrupole electric field, the PTSX device is composed of cylindrical electrodes of radius $r_{w}=10 \mathrm{~cm}$ that are sliced into four $90^{\circ}$ azimuthal sectors (Fig. 1). The central electrodes have length $2 L=2 \mathrm{~m}$, while the end electrodes are each $40 \mathrm{~cm}$ long. The trap confines nonneutral ion plasmas in the transverse direction by applying an oscillating voltage $\pm V_{0}(t)$ with frequency $f$ to the four sectors of the central electrode, creating a ponderomotive force that is directed transversely inwards. A DC voltage $+\hat{V}$, which provides an axial potential well to confine the charge bunch axially, is applied to the end electrodes. The PTSX device

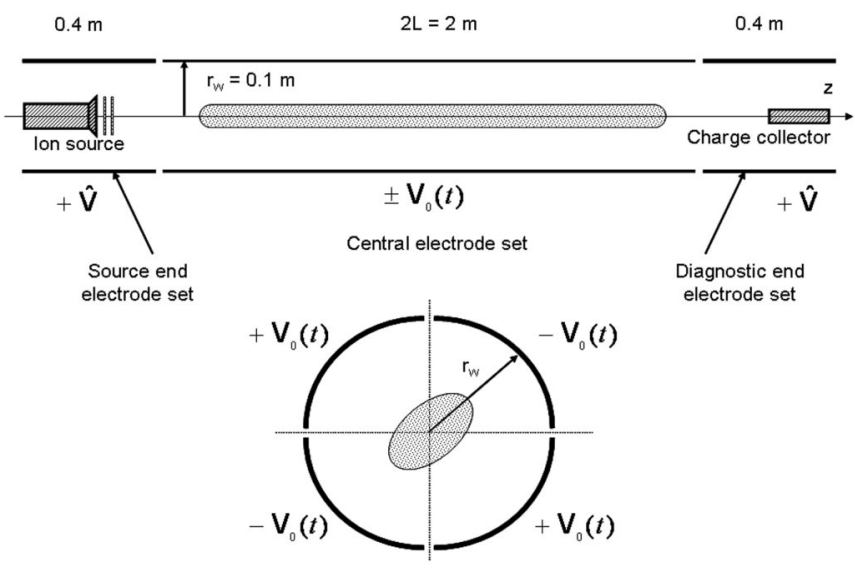

FIG. 1. Schematic of the PTSX device showing the quadrupole electrodes, ion source, and charge collector. 
manipulates the plasma using an inject-trap-dump cycle, and the one-component pure ion plasmas created in the trap are highly reproducible. During injection, the short electrodes on the source end are made to oscillate with the same voltage waveform as the central electrodes. The injection time is several milliseconds in order to allow cesium ions with several $\mathrm{eV}$ of kinetic energy to fill the trap. After injection is finished, the source end electrodes are switched from the fully oscillating voltage waveform to their static bias voltages. Finally, a long $(\sim 2 \mathrm{~m})$ onecomponent ion plasma column is trapped between the positively biased end electrodes. The typical operating pressure and ion number density in PTSX are $5 \times$ $10^{-9}$ Torr and $1 \times 10^{5} \mathrm{~cm}^{-3}$, respectively, and the plasma is collisionless to very good approximation.

\section{ANALYTICAL THEORY}

For simplicity in the theoretical analysis presented in this section, we assume that the beam states before and after the transition are both quasiequilibrium states, and the average effects of the quadrupole focusing field are described by an equivalent smooth-focusing force $[1,2]$. In equilibrium, radial force balance on a fluid element of beam ions with charge $q$ and mass $m$ in the smoothfocusing approximation is given by [1]

$$
\frac{\partial}{\partial r} P_{\perp b}(r)=-q n_{b}(r) \frac{\partial}{\partial r} \phi^{s}(r)-m \omega_{q}^{2} n_{b}(r) r,
$$

where $n_{b}(r)$ is the radial density profile, $\omega_{q}$ is the applied smooth-focusing frequency, and $r$ is the radial distance from the beam axis. Here, the space-charge potential $\phi^{s}(r)$ is determined self-consistently from Poisson's equation $r^{-1} \partial_{r}\left(r \partial_{r} \phi^{s}\right)=-q n_{b}(r) / \epsilon_{0}$, and $P_{\perp b}(r)=$ $n_{b}(r) m\left\langle v_{\perp}^{2}\right\rangle / 2$ is the perpendicular pressure profile, where $\epsilon_{0}$ is the permittivity of free space and the angular bracket $\langle\cdots\rangle$ denotes statistical average over the equilibrium distribution function in transverse velocity space. Manipulation of Eq. (1) gives the global radial force balance equation [1]

$$
m \omega_{q}^{2} R_{b}^{2}=2 \bar{T}_{\perp}+\frac{N_{b} q^{2}}{4 \pi \epsilon_{0}},
$$

where $N_{b}=2 \pi \int_{0}^{r_{w}} d r r n_{b}(r)$ is the line density, $R_{b}^{2}=$ $\left(2 \pi / N_{b}\right) \int_{0}^{r_{w}} d r r r^{2} n_{b}(r)$ is the mean-squared radius of the beam, and $\bar{T}_{\perp}$ is the effective transverse temperature defined by

$$
\bar{T}_{\perp}=\frac{2 \pi}{N_{b}} \int_{0}^{r_{w}} d r r \frac{1}{2} m\left\langle v_{\perp}^{2}\right\rangle n_{b}(r) .
$$

Note that the effective transverse temperature $\bar{T}_{\perp}$ measures the kinetic energy per particle averaged over the transverse phase space.

For an applied voltage waveform $V_{0}(t)=$ $V_{0 \max } \sin (2 \pi f t)$, the smooth-focusing frequency is given

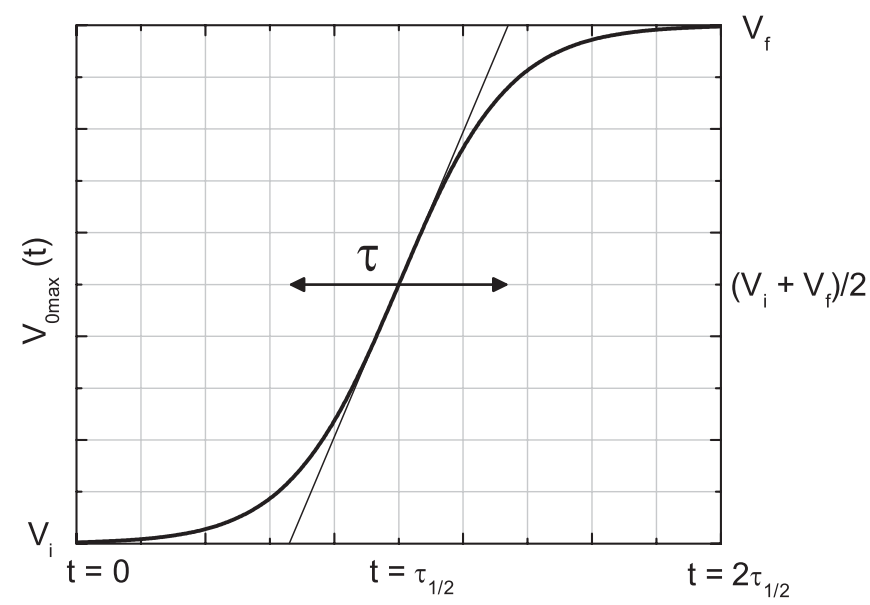

FIG. 2. Plot of voltage waveform amplitude $V_{0 \max }(t)$ used in the compression experiments. During the characteristic time scale $\tau$, about $80 \%$ of the transition is completed.

approximately by [12]

$$
\omega_{q}=\frac{8 q V_{0 \max }}{m \pi r_{w}^{2} f} \frac{1}{2 \sqrt{2} \pi} .
$$

By increasing or decreasing the voltage waveform amplitude $V_{0 \max }$ at fixed focusing frequency $f$, we can change $\omega_{q}$ accordingly. If $\omega_{q}$ is changed, a new quasiequilibrium state will be achieved satisfying the force balance equation (2) with new values of $R_{b}$ and $\bar{T}_{\perp}$. When $\omega_{q}$ is increased, the plasma is compressed; and when $\omega_{q}$ is decreased, the plasma expands. To describe transitions ranging from instantaneous changes to adiabatic changes, we adopt a simple model in which $V_{0 \max }$ varies in time according to

$$
V_{0 \max }(t)=V_{i}+\left(V_{f}-V_{i}\right)\left[\exp \left(\frac{\tau_{1 / 2}-t}{\tau / 4}\right)+1\right]^{-1},
$$

where $\tau_{1 / 2}$ is the time at which the transition is half complete, and $\tau$ is the characteristic time scale for the variation of $V_{0 \max }(t)$ from the initial amplitude $V_{i}$ to the final amplitude $V_{f}$ (Fig. 2). The number of lattice periods for transition can be defined as $N_{t}=\tau f$, where $N_{t}=0$ corresponds to an instantaneous change. We assume that the transition begins at $t=0$ when the beam is in an initial equilibrium state and well characterized, and is essentially complete by $t=2 \tau_{1 / 2}$. For continuity of the voltage waveform at $t=0$ and $t=2 \tau_{1 / 2}$, we require $\exp \left(4 \tau_{1 / 2} / \tau\right) \gg 1$.

\section{A. Instantaneous transition}

The analysis in this section makes use of total energy conservation discussed by Reiser [14]. When a beam is in a quasiequilibrium state, the average transverse kinetic energy per particle is identified with the effective transverse temperature as $E_{k}=\bar{T}_{\perp}$. Moreover, the average potential energy per particle associated with the external focusing force is calculated to be 


$$
E_{p}=\frac{2 \pi}{N_{b}} \int_{0}^{r_{w}} d r r \frac{1}{2} m \omega_{q}^{2} r^{2} n_{b}(r)=\frac{1}{2} m \omega_{q}^{2} R_{b}^{2},
$$

in the smooth-focusing approximation. The average electrostatic energy associated with the self-electric field is defined by $E_{e}=\left(2 \pi / N_{b}\right) \int_{0}^{r_{w}} d r r \frac{1}{2} \epsilon_{0}\left(-\partial_{r} \phi^{s}\right)^{2}$ [1]. For analytical simplicity, we assume that the beam has a uniform density profile $n_{b}(r)=\hat{n}_{b}=$ const for $0 \leq$ $r<\sqrt{2} R_{b}$. Then the electrostatic energy [1] can be calculated as $E_{e}=\left(N_{b} q^{2} / 4 \pi \epsilon_{0}\right)\left[\ln \left(r_{w} / \sqrt{2} R_{b}\right)+1 / 4\right]$, where $r_{w}$ is the radius of the perfectly conducting wall. Therefore, the total energy per particle, $E=E_{k}+E_{p}+$ $E_{e}$, for a beam with uniform density profile can be expressed as

$$
E=m \omega_{q}^{2} R_{b}^{2}+\frac{N_{b} q^{2}}{4 \pi \epsilon_{0}}\left[\ln \left(\frac{r_{w}}{\sqrt{2} R_{b}}\right)-\frac{1}{4}\right],
$$

where use has been made of the global force balance equation (2) to eliminate $\bar{T}_{\perp}$.

For an instantaneous change of focusing field strength from $\omega_{q i}$ to $\omega_{q f}$, there will be an energy difference between initial and final equilibrium states given by $\Delta E=$ $m\left(\omega_{q f}^{2}-\omega_{q i}^{2}\right) R_{b i}^{2} / 2$, where $R_{b i}$ is the rms radius of the initial beam, which is assumed to remain constant at the instant of transition. Note that $\Delta E>0$ for compression, while $\Delta E<0$ for expansion. This energy difference represents the free energy that can be redistributed by nonlinear space-charge forces or instabilities [2]. If the beam relaxes from an initial state with energy $E_{i}$ into a final quasiequilibrium state with energy $E_{f}=E_{i}+\Delta E$, we obtain the transcendental equation for the final rms radius $R_{b f}$,

$$
\left(\frac{R_{b f}}{R_{b i}}\right)^{2}-\frac{K_{b}}{2 \omega_{q f}^{2} R_{b i}^{2}} \ln \left(\frac{R_{b f}}{R_{b i}}\right)-\frac{1}{2}\left(1+\frac{\omega_{q i}^{2}}{\omega_{q f}^{2}}\right)=0,
$$

where $K_{b}=2 N_{b} q^{2} / 4 \pi \epsilon_{0} m$ is the effective self-field perveance, and the line density $N_{b}$ is assumed to remain constant during the transition. Redistribution of the free energy $\Delta E$ usually introduces an emittance growth $[2,3]$. Because there is often a large mismatch after an instantaneous transition, this emittance growth typically occurs on the time scale of the envelope oscillations, and is associated with the formation of a low-density halo region [1-3]. Since the formation of the halo is not included in the present simplified theoretical model, the final rms radius $R_{b f}$ measured in experiments with a significant halo contribution during an instantaneous transition is usually larger than the analytical estimate in Eq. (8) [for example, see Fig. 9(a) in Sec. IV of this paper].

\section{B. Adiabatic transition}

Beams in an ideal equilibrium do not experience emittance growth. However, beams are rarely in an ideal equi- librium, and any changes in the focusing system and nonuniformities in charge density can produce changes in the beam distribution, usually accompanied by emittance growth [3]. Transverse compression also induces emittance growth. However, we can minimize the emittance growth by compressing the beam very slowly. For a quantitative description of the adiabaticity of the compression process, we make use of the following equation that describes the evolution of the rms radius of a long charge bunch with uniform density profile $[1,2,5]$ :

$$
\frac{d^{2}}{d t^{2}} R_{b}+\left(\omega_{q}^{2}(t)-\frac{K_{b}}{2 R_{b}^{2}}\right) R_{b}=\frac{\epsilon^{2}(t)}{4 R_{b}^{3}}
$$

Here, the smooth-focusing frequency $\omega_{q}(t)$ evolves in time according to Eqs. (4) and (5), and $\epsilon(t)=2 R_{b}\left[\left(2 \bar{T}_{\perp} / m\right)-\right.$ $\left.\left(d R_{b} / d t\right)^{2}\right]^{1 / 2}$ is the unnormalized transverse emittance defined in the beam frame. To describe the time evolution of the transverse emittance self-consistently, we need to solve the nonlinear Vlasov-Maxwell equation numerically. However, for the case of an adiabatic transition in $\omega_{q}(t)$, we can assume $\epsilon(t) \approx 2 R_{b}\left(2 \bar{T}_{\perp} / m\right)^{1 / 2}=$ const [5]. If we change $\omega_{q}(t)$ slowly, then there is a small-amplitude perturbation around the quasiequilibrium radius $\bar{R}_{b}=\left[\left(K_{b}+\right.\right.$ $\left.\left.\sqrt{K_{b}^{2}+4 \epsilon^{2} \omega_{q}^{2}}\right) / 4 \omega_{q}^{2}\right]^{1 / 2}$, with oscillation frequency given by $\omega_{b}=2 \omega_{q}\left(1-K_{b} / 4 \bar{R}_{b}^{2} \omega_{q}^{2}\right)^{1 / 2}$. As long as the characteristic transition time $\tau$ is much longer than $\omega_{b}^{-1}$, the beam mismatch induced by changing $\omega_{q}$ remains small [5]. Therefore, the condition for adiabatic transition is given by

$$
\frac{N_{t}}{f} \min \left[2 \omega_{q}\left(1-K_{b} / 4 \bar{R}_{b}^{2} \omega_{q}^{2}\right)^{1 / 2}\right]>1 .
$$

It is evident from Eq. (10) that for the case of beam expansion $\left(\omega_{q f} / \omega_{q i}<1\right)$ a larger number $N_{t}$ of lattice periods is required for the adiabatic transition to occur. If the beam relaxes adiabatically from an initial state with $\epsilon_{i}=2 R_{b i}\left(2 \bar{T}_{\perp i} / m\right)^{1 / 2}$ to a final state with $\epsilon_{f}=$ $2 R_{b f}\left(2 \bar{T}_{\perp f} / m\right)^{1 / 2}$, then by assuming $\epsilon_{i} \simeq \epsilon_{f}$ and making use of Eq. (2) we obtain the simple algebraic equation for the final rms radius $R_{b f}$,

$$
\left(\frac{R_{b f}}{R_{b i}}\right)^{4}-\frac{K_{b}}{2 \omega_{q f}^{2} R_{b i}^{2}}\left[\left(\frac{R_{b f}}{R_{b i}}\right)^{2}-1\right]-\frac{\omega_{q i}^{2}}{\omega_{q f}^{2}}=0,
$$

where it is assumed that $K_{b}$ (or equivalently $N_{b}$ ) remains constant during the transition.

\section{EXPERIMENTAL RESULTS}

The initial charge bunch for the compression experiments is trapped in the PTSX device with a sinusoidal voltage waveform with $f=60 \mathrm{kHz}$ and $V_{0 \max }=$ $150.4 \mathrm{~V}$, which corresponds to $\omega_{q}=52.2 \mathrm{kHz}$ and 
smooth-focusing vacuum phase advance $\sigma_{v}^{s f}=\omega_{q} / f=$ $49.8^{\circ}$. Ion injection into the trap has been optimized in order that the initial beam is both well matched to the focusing lattice and well characterized [15]. The initial beam has normalized intensity $s=\omega_{p}^{2}(0) / 2 \omega_{q}^{2} \sim 0.22$, and its density profile is nearly Gaussian with $R_{b}=$ $0.85 \mathrm{~cm}$ and $\bar{T}_{\perp}=0.13 \mathrm{eV}$ [Fig. 3(a)]. Here, $\omega_{p}^{2}(r)=$ $n_{b}(r) q^{2} / \epsilon_{0} m$ is the plasma frequency squared. By changing the amplitude of the voltage waveform at fixed focusing frequency, various instantaneous and adiabatic transitions are applied to the charge bunch. The total duration of the transition $2 \tau_{1 / 2}$ is typically $1 \mathrm{msec}$, which is equivalent to 60 lattice periods for $f=60 \mathrm{kHz}$. The radial charge profile $Q(r)$, which is proportional to the number density $n_{b}(r)$, is measured by averaging the signal at each radial position over 20 repeated measurements. The size of the charge collector aperture $r_{a}$ and an estimate of the length of the trapped plasma $L_{p}$ are then used to calculate $n_{b}(r) \approx Q(r) / q \pi r_{a}^{2} L_{p}$. For example, the onaxis charge $Q(0)$ prior to the transition in Figs. 3(a) and 4 corresponds to an on-axis density $n_{b}(0)$ of $0.83 \times$
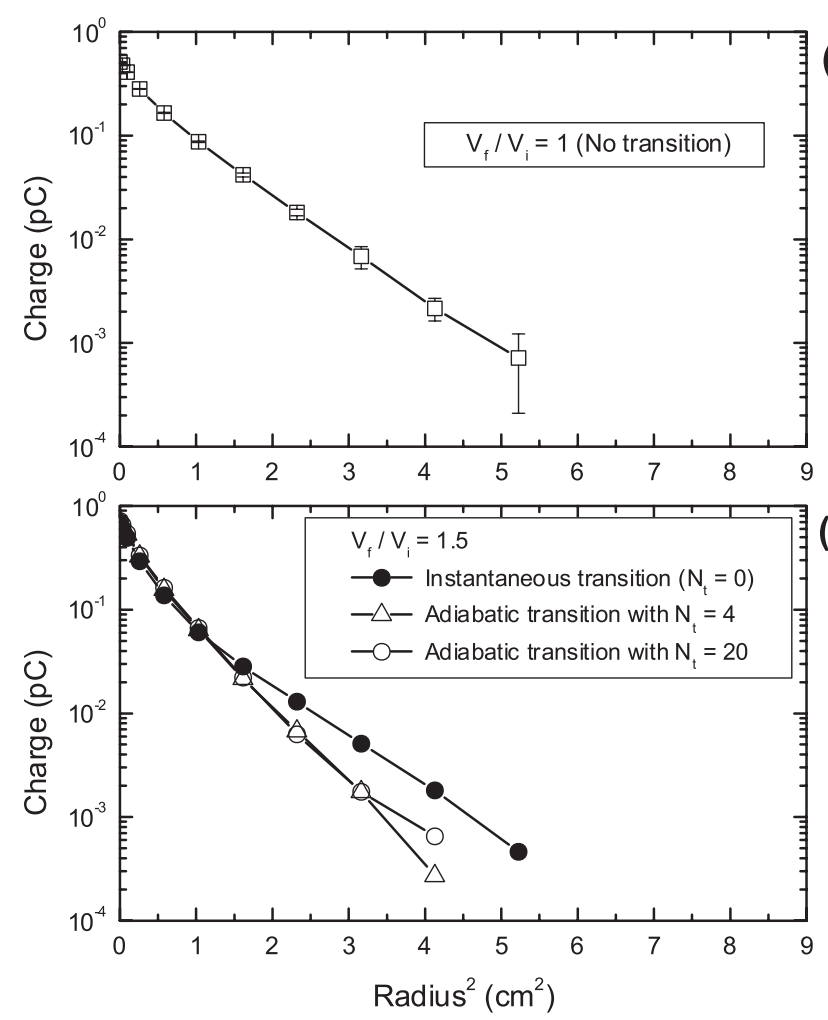

(a)

FIG. 3. Measured radial beam profiles (a) prior to transitions and (b) after transitions with $V_{f} / V_{i}=1.5$. The transitions are made instantaneously (solid circles), adiabatically with $N_{t}=4$ (open triangles), and adiabatically with $N_{t}=20$ (open circles). The initial smooth-focusing vacuum phase advance $\sigma_{v i}^{s f}$ is $49.8^{\circ}$, and the applied focusing frequency $f$ is fixed at $60 \mathrm{kHz}$ during the transitions. A straight line in the $\log$ versus $r^{2}$ plot indicates that the radial profile is a Gaussian function of $r$.
$10^{5} \mathrm{~cm}^{-3}$. Since the trapped plasmas are highly reproducible and the offset errors in the charge collector system are typically less than $1 \mathrm{fC}$, the relative errors in the calculation of low-order moments of the particle distribution (such as line density, rms radius, and emittance) are typically a few percent. Typical standard errors in the radial profile measurement are also shown in Fig. 3(a).

In Fig. 4, scans of the number $N_{t}$ of lattice periods for the compression transition reveal that, for voltage amplitude increases of $50 \%$ and $90 \%$, the compression leads to an increase in the on-axis charge that saturates after the transition is made in several lattice periods. There is no extra benefit in adiabatic compression by making the transition more gradual than approximately four lattice periods. Indeed, the condition for adiabaticity, $\tau \omega_{b} \approx 6.9>1$, is well satisfied for compressions with $N_{t}=4$. It is interesting to note that for $N_{t}=0$ (instantaneous transition), due to the beam loss and emittance growth associated with the large beam mismatch, the on-axis charge after the instantaneous transition with $V_{f} / V_{i}=1.9$ becomes smaller than for the case with $V_{f} / V_{i}=1.5$. Figure 3 indicates that the measured radial profiles for adiabatic transitions with $N_{t}=4$ and $N_{t}=20$ are almost identical, whereas for the instantaneous case $\left(N_{t}=0\right)$, the radial profile broadens considerably. Another example that illustrates this point is presented in Fig. 5 by solving Eq. (9) numerically. For an adiabatic compression with $V_{f} / V_{i}=$ 1.5 , it is evident that $N_{t}=4$ constitutes enough lattice periods to avoid mismatch oscillations, as expected from the experimental data. However, to make certain that the

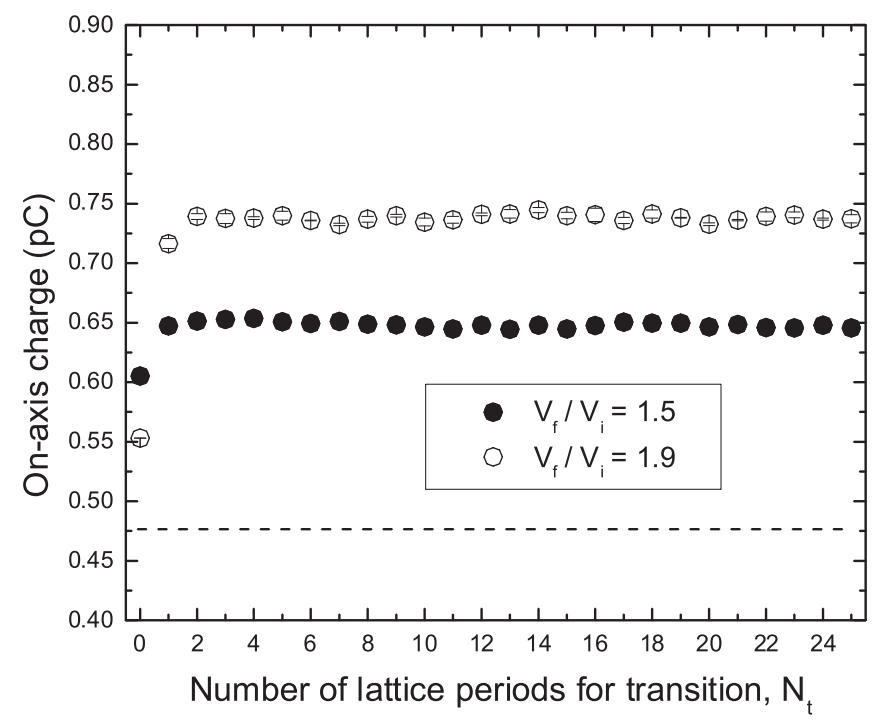

FIG. 4. Measured on-axis charge dependence on the number $N_{t}$ of lattice periods for transitions with $V_{f} / V_{i}=1.5$ (solid circles) and $V_{f} / V_{i}=1.9$ (open circles) for $\sigma_{v i}^{s f}=49.8^{\circ}$ and $f=$ $60 \mathrm{kHz} . N_{t}=0$ corresponds to an instantaneous transition. In Fig. 4, the on-axis charge prior to the transition is $0.476 \mathrm{pC}$, which is indicated by dashed line. 


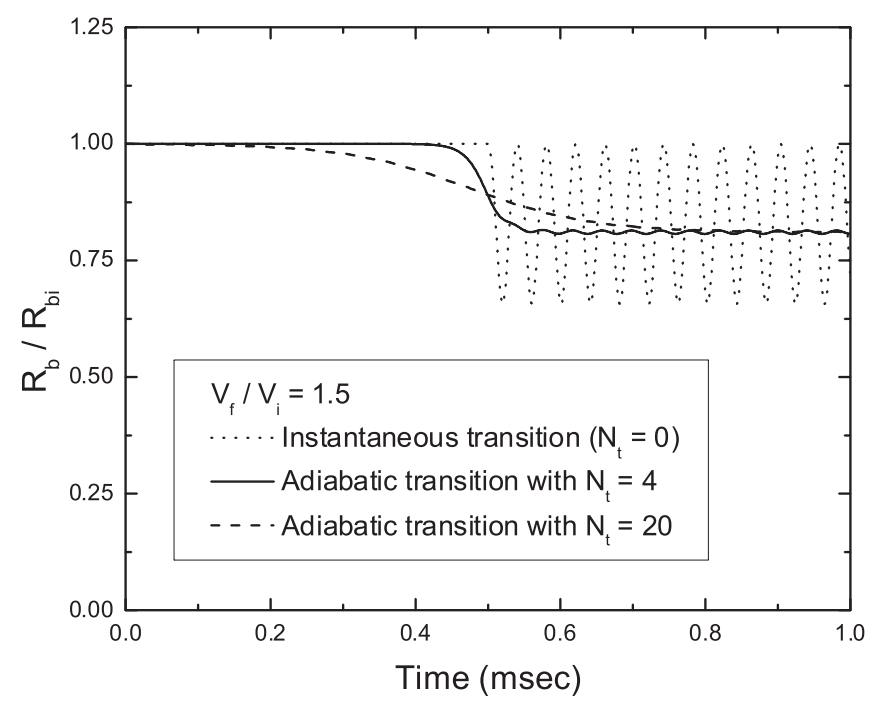

FIG. 5. Numerical solutions to the envelope Eq. (9) with $\epsilon(t)=$ const. Evolution of the rms beam radius during an instantaneous transition (dotted line), adiabatic transition with $N_{t}=4$ (solid line), and adiabatic transition with $N_{t}=20$ (dashed line). Here, $V_{f} / V_{i}=1.5, \sigma_{v i}^{s f}$ is $49.8^{\circ}$, and $f=60 \mathrm{kHz}$.

transitions are sufficiently gradual, we have performed the subsequent adiabatic transition experiments with $N_{t}=20$ for compression, and $N_{t}=40$ for expansion. [As noted earlier in relation to Eq. (10), larger values of $N_{t}$ are required for the case of expansion.] If $N_{t}>40$, then discontinuities in the voltage amplitude at the start point and end point become larger than 5\%, which can be another source of mismatch.

In Fig. 6, the ratio of the final to initial on-axis beam density $n_{b f}(0) / n_{b i}(0)$ is estimated analytically and measured experimentally. The transition is made either instantaneously or adiabatically, and the ratio of the final to initial voltage amplitude, $V_{f} / V_{i}$, is scanned from 0.1 to 2.2. Since the applied focusing frequency is fixed, we have $V_{f} / V_{i}=\omega_{q f} / \omega_{q i}=\sigma_{v f}^{s f} / \sigma_{v i}^{s f}$. Except for the lower $\left(V_{f} / V_{i}<0.6\right)$ and upper $\left(V_{f} / V_{i}>1.6\right)$ ranges, the experimental data are in relatively good agreement with the analytical estimates. In Fig. 6(a), the theoretical estimates are made by using Eqs. (8) and (11). When it is assumed that the transverse emittance is approximately constant during the adiabatic transition [5], the on-axis beam density increases approximately linearly according to the increase in the voltage amplitude. In Fig. 6(b), the on-axis beam density (or equivalently, the on-axis charge) is measured by opening the diagnostic-end electrodes immediately after the transition is complete. Since it takes about $2 \mathrm{msec}$ to dump all of the trapped plasma to the charge collector, the measured signal for a single inject-trap-dump cycle is necessarily averaged over about 120 lattice oscillation periods for $f=60 \mathrm{kHz}$. It is clear that in the range $0.9 \leq V_{f} / V_{i} \leq 1.2$, there is no noticeable difference in the

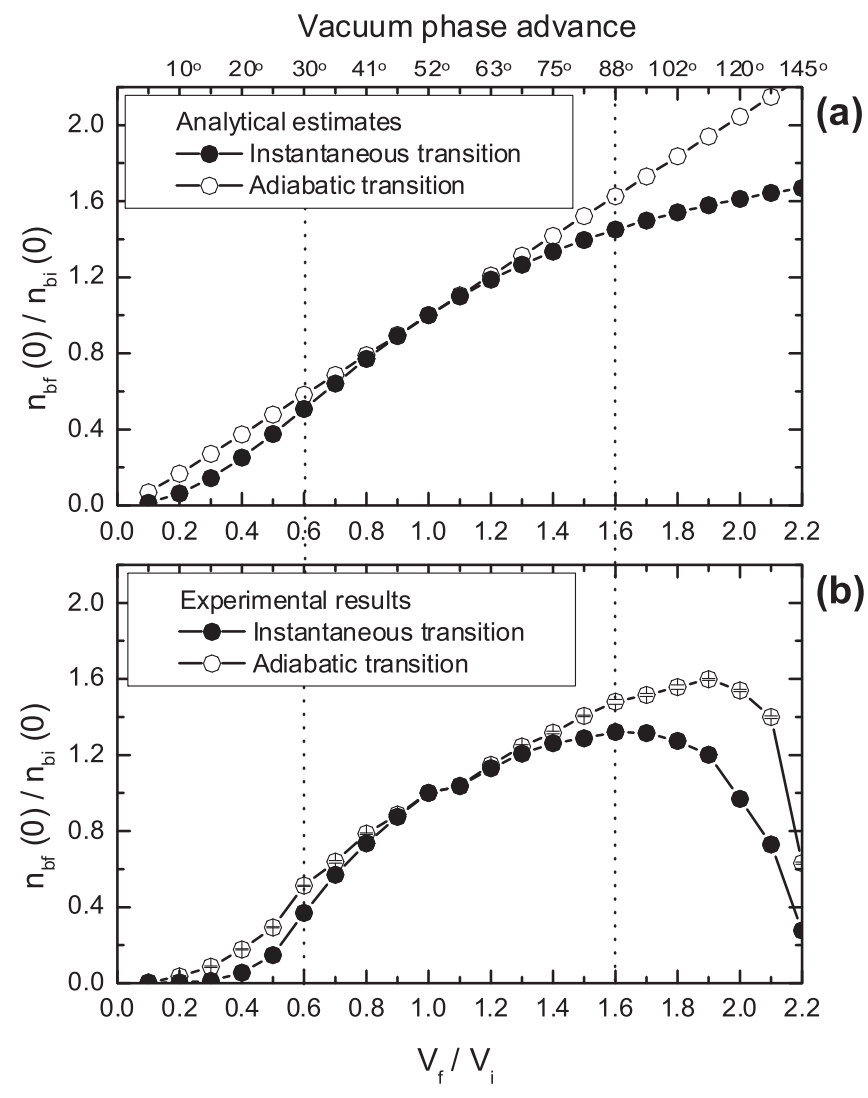

FIG. 6. Plots of the ratio of final to initial on-axis beam density $n_{b f}(0) / n_{b i}(0)$ for different values of $V_{f} / V_{i}$ with $f=60 \mathrm{kHz}$. The values $n_{b f}(0) / n_{b i}(0)$ are either (a) estimated from analytical theory, or (b) measured experimentally. Here, the initial vacuum phase advance $\sigma_{v i}$ is $52^{\circ}$, which corresponds to $\sigma_{v i}^{s f}=49.8^{\circ}$. Both instantaneous (solid circles) and adiabatic (open circles) transitions are considered.

on-axis beam density between the instantaneous and adiabatic cases. In fact, the measured radial profiles for the instantaneous and adiabatic compression cases, for $V_{f} / V_{i}=1.2$, are almost indistinguishable [Fig. 7(a)]. This suggests that modest changes ( $\lesssim 20 \%$ ) in the focusing field strength may be made abruptly, without the need for a lengthy gradual-transition region. For example, if we increase the voltage amplitude by $20 \%$ for each cycle, then after four cycles we find $V_{f} / V_{i}=(1.2)^{4} \approx 2.1$. This may explain why only several lattice periods are adequate for adiabatic compression when $V_{f} / V_{i}=1.9$ in Fig. 4.

It is also interesting to note, for the adiabatic compression case, that the envelope instability [1,2] can affect the on-axis plasma density when $V_{f} / V_{i}>1.6$. We define vacuum phase advance $\sigma_{v}$ as the phase advance of a particle oscillating in a quadrupole focusing field in the absence of space-charge force $[1,2,16]$. For $V_{f} / V_{i}=1.6, \sigma_{v}$ is approximately $88^{\circ}$. Therefore, when the initial charge bunch is compressed by more than a $60 \%$ increase in the voltage amplitude, the charge bunch enters the unstable parameter 

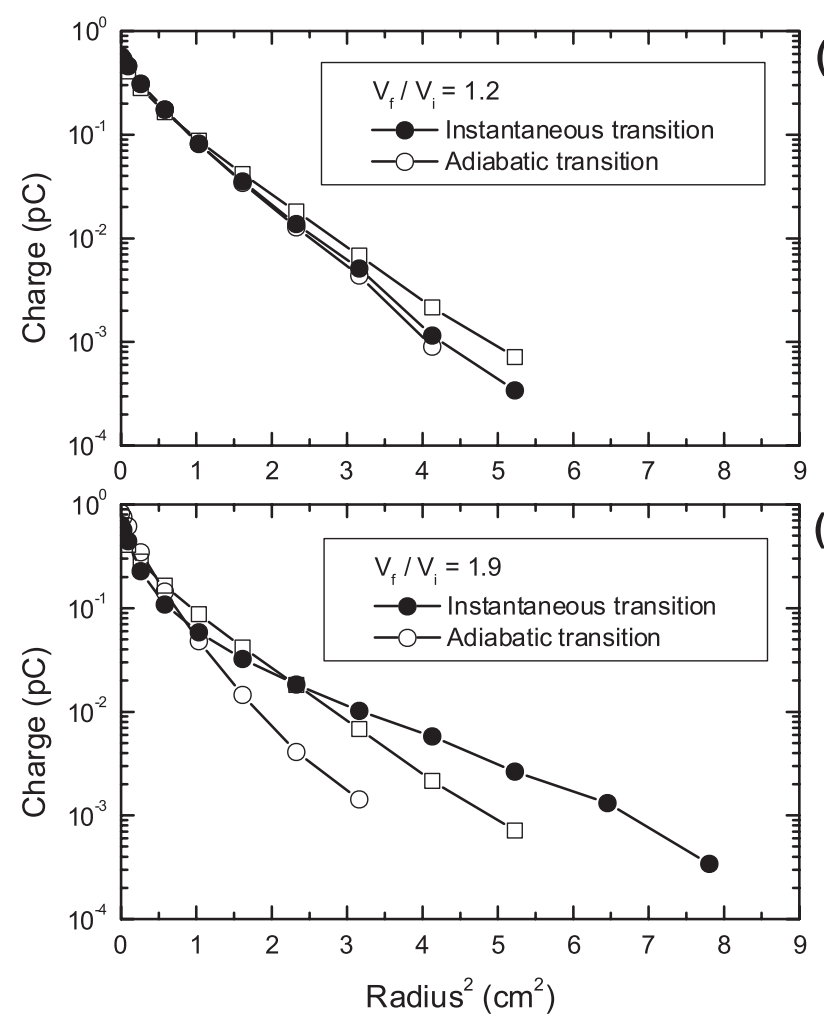

(b)

(a)

FIG. 7. Measured radial beam profiles after transitions with (a) $V_{f} / V_{i}=1.2$, and (b) $V_{f} / V_{i}=1.9$ for $\sigma_{v i}^{s f}=49.8^{\circ}$ and $f=$ $60 \mathrm{kHz}$. The transitions are made either instantaneously (solid circles), or adiabatically with $N_{t}=20$ (open circles). The squares in Fig. 7 correspond to the radial profile for the case where $V_{f}=V_{i}$ (no transition). A straight line in the log versus $r^{2}$ plot indicates that the radial profile is a Gaussian function of $r$.

region for the envelope instability $\left(\sigma_{v}>90^{\circ}\right)$ [2]. However, for the moderate space-charge-density beams $(s=0.2-0.3)$ considered in the present study, the tune depression $\nu / \nu_{0} \approx(1-s)^{1 / 2}$ is approximately $0.84-$ 0.89 , and the instability band around $\sigma_{v}=90^{\circ}$ is usually very narrow [16]. Therefore, if the beam is compressed in such a way that the beam passes through the instability band quickly (for example, in several lattice periods for the present study), then we can minimize the emittance growth associated with the excitation of the envelope instability. In the experimental results presented in Fig. 6(b), no detrimental beam degradation is observed even when the final vacuum phase advance $\sigma_{v f}$ becomes larger than $90^{\circ}$ (or equivalently $V_{f} / V_{i}>1.6$ ). Nonetheless, when the initial beam is further compressed with $V_{f} / V_{i} \gtrsim 2.2$, then $\sigma_{v} \rightarrow$ $180^{\circ}$, and we begin to lose confinement of the beam particles.

Plots of the ratio of final to initial line density $N_{b f} / N_{b i}$ for different values of $V_{f} / V_{i}$ are shown in Fig. 8, from which we can verify particle number conservation in the transition experiments. As expected, for the case of transverse compression, adiabatic compression is more effec-

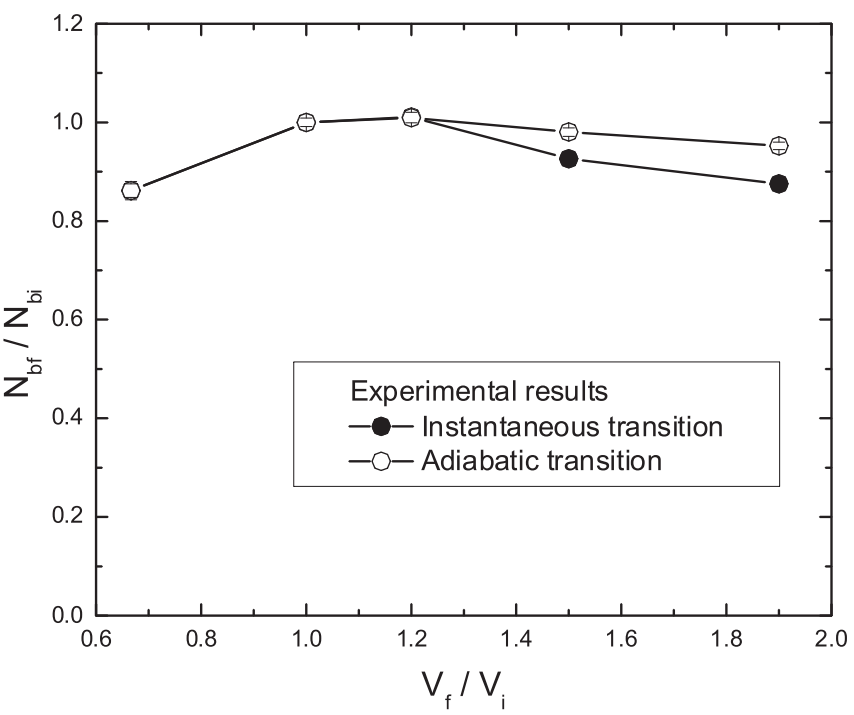

FIG. 8. Plots of the ratio of final to initial line density $N_{b f} / N_{b i}$ for different values of $V_{f} / V_{i}$. The values of $N_{b f} / N_{b i}$ are measured from experiments with instantaneous transitions (solid circles), and adiabatic transitions (open circles). Here, $\sigma_{v i}^{s f}=$ $49.8^{\circ}$ and $f=60 \mathrm{kHz}$.
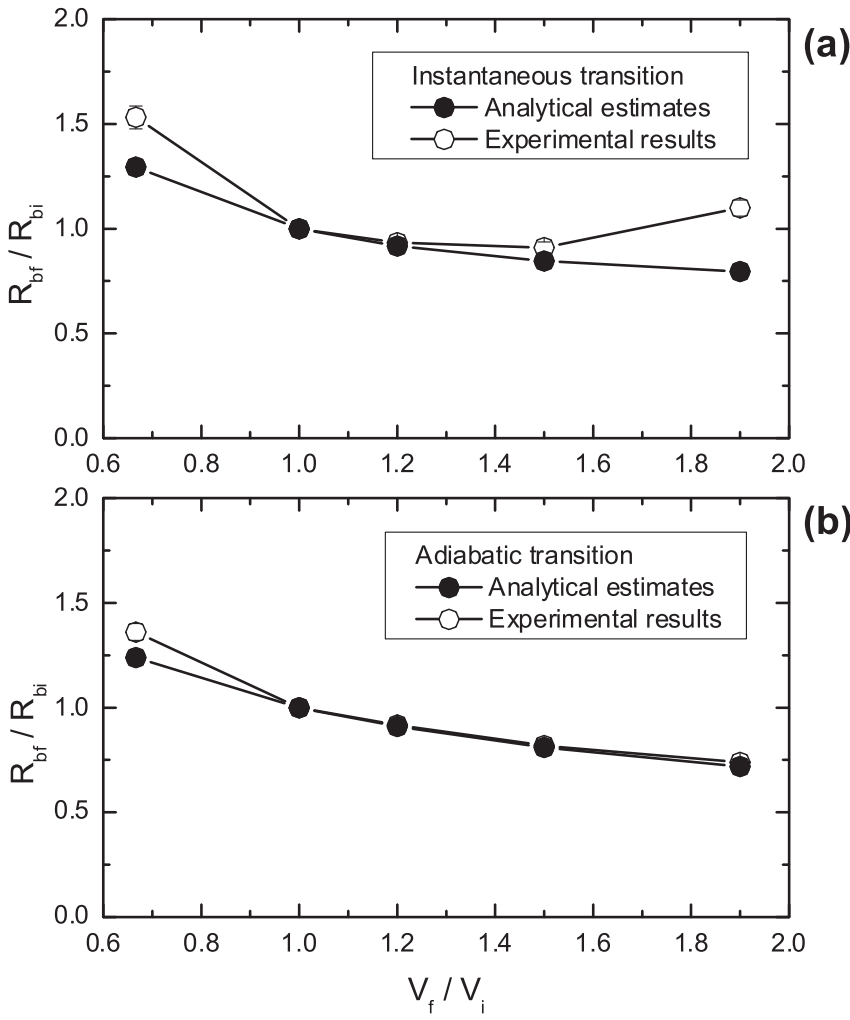

FIG. 9. Plots of the ratio of final to initial rms radius $R_{b f} / R_{b i}$ for different values of $V_{f} / V_{i}$. The values of $R_{b f} / R_{b i}$ are either estimated based on analytical theory (solid circles), or inferred from experimental data (open circles). The two cases correspond to (a) instantaneous transitions, and (b) adiabatic transitions. Here, $\sigma_{v i}^{s f}=49.8^{\circ}$ and $f=60 \mathrm{kHz}$. 
tive in minimizing particle losses. Considering the detection limit ( $\sim 1 \mathrm{fC}$ ) of the charge collector system, particle loss can be interpreted as the formation of a tenuous halo at large radius. In Fig. 9, we plot the ratio of final to initial rms radius $R_{b f} / R_{b i}$ for different values of $V_{f} / V_{i}$. The values of final rms beam radius are either estimated based on analytical theory, or calculated from the measured radial profiles. For the case of adiabatic compression, the experimentally determined rms radius is in good agreement with the analytical estimate [Fig. 9(b)]. This is mainly because mismatch oscillations are minimized during adiabatic compression. On the other hand, an instantaneous transition induces mismatch oscillations, which causes the measured radial profile to have a superGaussian tail [see, for example, Fig. 7(b)]. Hence, the experimentally determined rms radius for the case of instantaneous transition is somewhat larger than the analytical estimate [Fig. 9(a)]. The ratios of final to initial transverse emittance $\epsilon_{f} / \epsilon_{i}$ are illustrated in Fig. 10 for different values of $V_{f} / V_{i}$. For the experimental results, the emittance is inferred from Eq. (2) and the measured radial profile, which determines $N_{b}$ and $R_{b}^{2}$. It is remarkable that the experimental data for adiabatic compression show that the emittance remains almost constant during the compres-
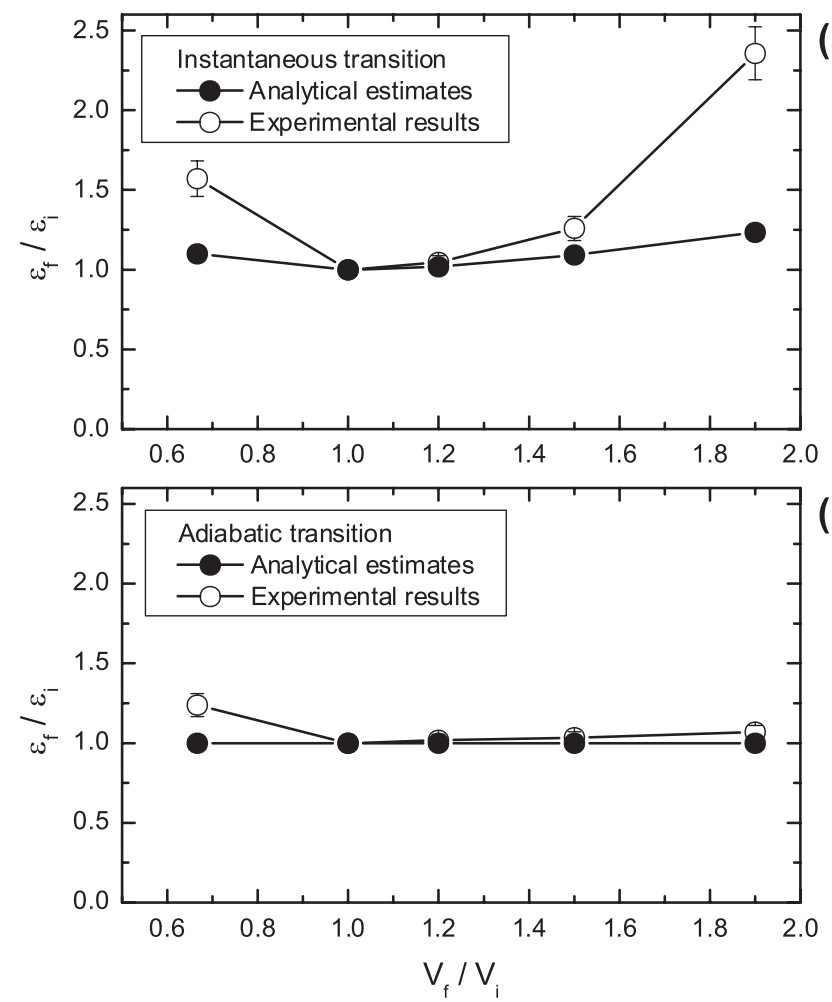

FIG. 10. Plots of the ratio of final to initial transverse emittance $\epsilon_{f} / \epsilon_{i}$ for different values of $V_{f} / V_{i}$. The values of $\epsilon_{f} / \epsilon_{i}$ are either estimated based on analytical theory (solid circles), or inferred from experimental data (open circles). The two cases correspond to (a) instantaneous transitions, and (b) adiabatic transitions. Here, $\sigma_{v i}^{s f}=49.8^{\circ}$ and $f=60 \mathrm{kHz}$. sion process, which is approximately consistent with the analytical estimate. When the final focusing strength is too small or too large, the rms mismatch induces a significant emittance growth for the instantaneous case, as expected. For the case with $V_{f} / V_{i}=1.9$, the emittance more than doubles. In this case, an adiabatic transition has a large advantage over an instantaneous transition in obtaining high on-axis density, and minimizing the emittance growth. While an instantaneous transition leads to a radial profile that exhibits a broad halo region, an adiabatic transition results in a radial profile that is nearly Gaussian [Fig. 7(b)]. Note that a straight line in the log versus $r^{2}$ plot indicates that the radial profile is a Gaussian function of $r$.

In contrast, it should be noted from Figs. 8 and 10 that, for the case of expansion $\left(\omega_{q f} / \omega_{q i}<1\right)$, there is no distinct advantage provided by an adiabatic transition. This is because of various nonideal effects in the PTSX device, such as the 3D end effects caused when the beam pulse reflects from the DC potential, and by higher-order corrections to the quadrupole focusing field in the off-axis region [11], both of which become strong effects when the beam radius is sufficiently large.

\section{NUMERICAL SIMULATIONS WITH THE WARP PARTICLE-IN-CELL CODE}

In this section, we present numerical simulation results using the two-dimensional version of the WARP electrostatic particle-in-cell code [17] for the analysis of experimental data in regimes where there are significant departures from simple analytical estimates. The WARP code describes the beam's self-consistent response to an alternating-gradient quadrupole focusing field with timevarying amplitude. To describe the effective emittance of such a pulsating beam, we use the average transverse emittance defined by $\epsilon=\left(\epsilon_{x} \epsilon_{y}\right)^{1 / 2}$, where $\epsilon_{x}=\left(\left\langle x^{2}\right\rangle \times\right.$ $\left.\left\langle\dot{x}^{2}\right\rangle-\langle x \dot{x}\rangle^{2}\right)^{1 / 2}$ and $\epsilon_{y}=\left(\left\langle y^{2}\right\rangle\left\langle\dot{y}^{2}\right\rangle-\langle y \dot{y}\rangle^{2}\right)^{1 / 2}$ [5]. The simulation results in Figs. 11 and 12 show the normalized on-axis density $n_{b}(0) / n_{b i}(0)$, and the normalized average transverse emittance $\epsilon / \epsilon_{i}$ as functions of time. Simulation parameters for loading the initial particle distribution have been chosen in such a way that the normalized intensity parameter $s$ and initial transverse emittance $\epsilon_{i}$ are close to the measured values of the initial beam parameters in the experiments. By considering a transition time of $1 \mathrm{msec}$, and a dumping time of $2 \mathrm{msec}$, we perform simulations extending to $3 \mathrm{msec}$ after the initial quasiequilibrium state is formed.

The simulation results shown in Fig. 11 clearly indicate that instantaneous transitions introduce significant mismatch oscillations and emittance growth. The emittance increase, $\Delta \epsilon / \epsilon_{i}=\left(\epsilon-\epsilon_{i}\right) / \epsilon_{i}$, is $33 \%$ for $V_{f} / V_{i}=1 / 1.5$, and $250 \%$ for $V_{f} / V_{i}=1.9$, which are in much better agreement with the experimental data than the simple 

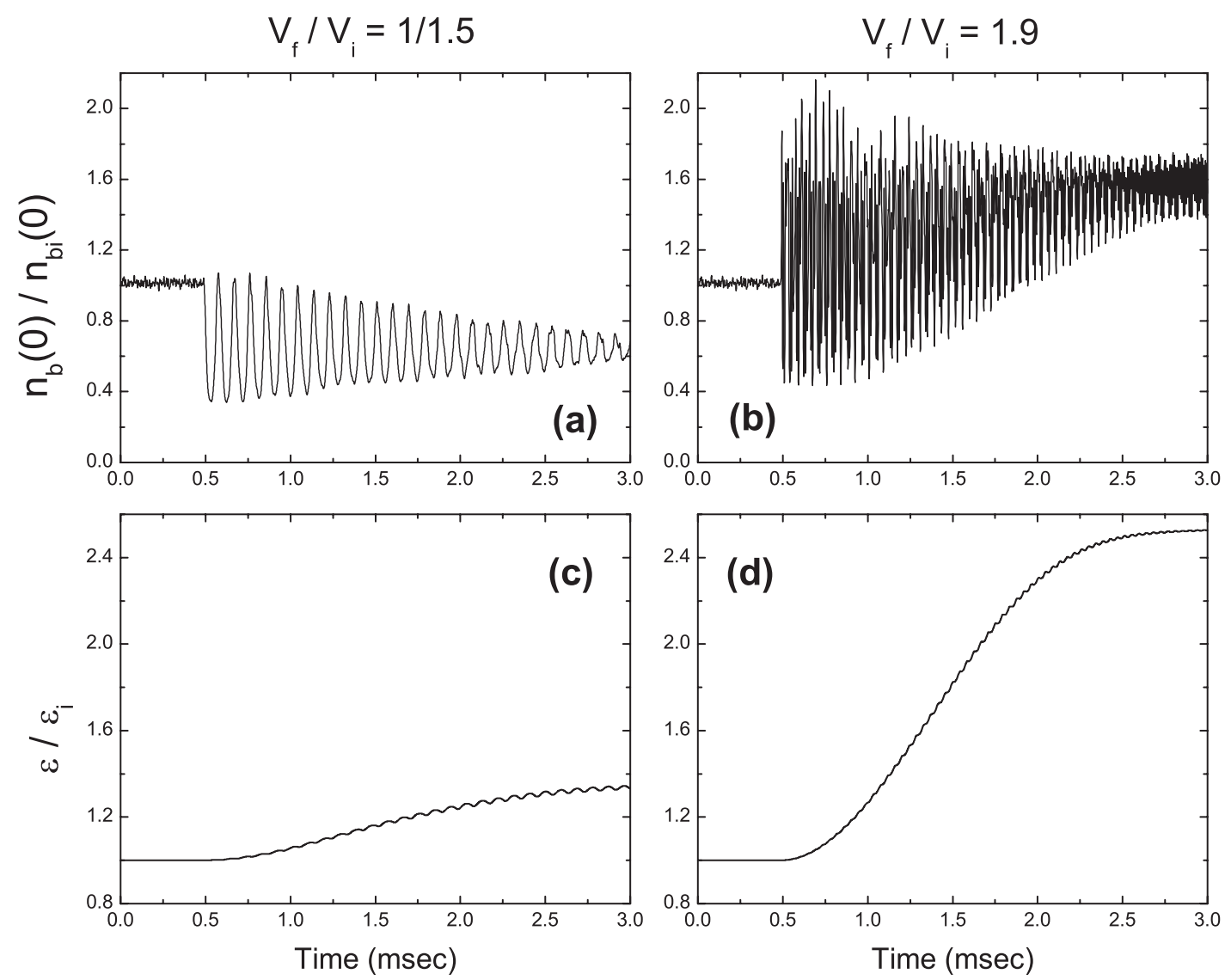

FIG. 11. 2D WARP simulations for an alternating-gradient quadrupole lattice. The evolution of the normalized on-axis density $n_{b}(0) / n_{b i}(0)$ and normalized transverse emittance $\epsilon / \epsilon_{i}$ are shown during an instantaneous transition process with $\sigma_{v i}^{s f}=49.8^{\circ}$ and $f=60 \mathrm{kHz}$. Frames (a) and (c) correspond to the case with $V_{f} / V_{i}=1 / 1.5$, and frames (b) and (d) correspond to the case with $V_{f} / V_{i}=1.9$.

analytical estimates in Sec. IV [see Fig. 10(a)], where there is no consideration of halo particle generation. For example, the emittance increase for $V_{f} / V_{i}=1.9$ in Fig. 10(a) is $25 \%$ based on the analytical estimate, and $240 \%$ from the actual measurement. Because of the large mismatch, significant oscillations in the on-axis plasma density last more than $2 \mathrm{msec}$ after the abrupt changes are made at $t=0.5 \mathrm{msec}$. Hence, the measured signals of the on-axis density can be interpreted as the time-averaged values of the on-axis density oscillations during the dumping process. Note that the frequency of the on-axis density oscillations observed in Fig. 11(a) is easily identified with the breathing mode frequency $\left(\omega_{b} \approx 67.9 \mathrm{kHz}\right)$.

Simulation results for an adiabatic compression case with $V_{f} / V_{i}=1.9$ are shown in Figs. 12(b) and 12(d) for $N_{t}=20$. As expected from the analytical estimates and experimental results, adiabatic compression assures that the final beam remains well matched, and the emittance growth during the transition is minimized $\left(\Delta \epsilon / \epsilon_{i}<0.5 \%\right)$. In the simulation results reported previously [18], it was shown that the adiabatic compression with $N_{t}=4$ also assures that the final beam stays well matched, which is consistent with the earlier discussions in the present paper. However, for the case of adiabatic expansion with $V_{f} / V_{i}=$ $1 / 1.5$, even the numerical simulations do not reproduce the experimental results [compare Figs. 10(b) and 12(c)]. As mentioned earlier in Sec. IV of this paper, the enhanced emittance growth observed in the expansion experiments is likely due to nonideal (e.g. 3D) effects [11], which are not considered in the two-dimensional version of the WARP code used in the present study. In contrast to the instantaneous transition cases, the on-axis densities relax almost immediately after the adiabatic transitions are complete at $t=1 \mathrm{msec}$. The final normalized on-axis density, $n_{b f}(0) / n_{b i}(0)$, is about 0.65 for $V_{f} / V_{i}=1 / 1.5$, and about 1.8 for $V_{f} / V_{i}=1.9$, which are slightly less than the analytical estimates in Fig. 6. Note that the on-axis density oscillations observed in Fig. 12(b) has a frequency of $120 \mathrm{kHz}$, which is twice the external focusing frequency $f$. This oscillation is a result of the pulsating motion of the beam envelope, and its amplitude increases considerably when the beam is compressed. 

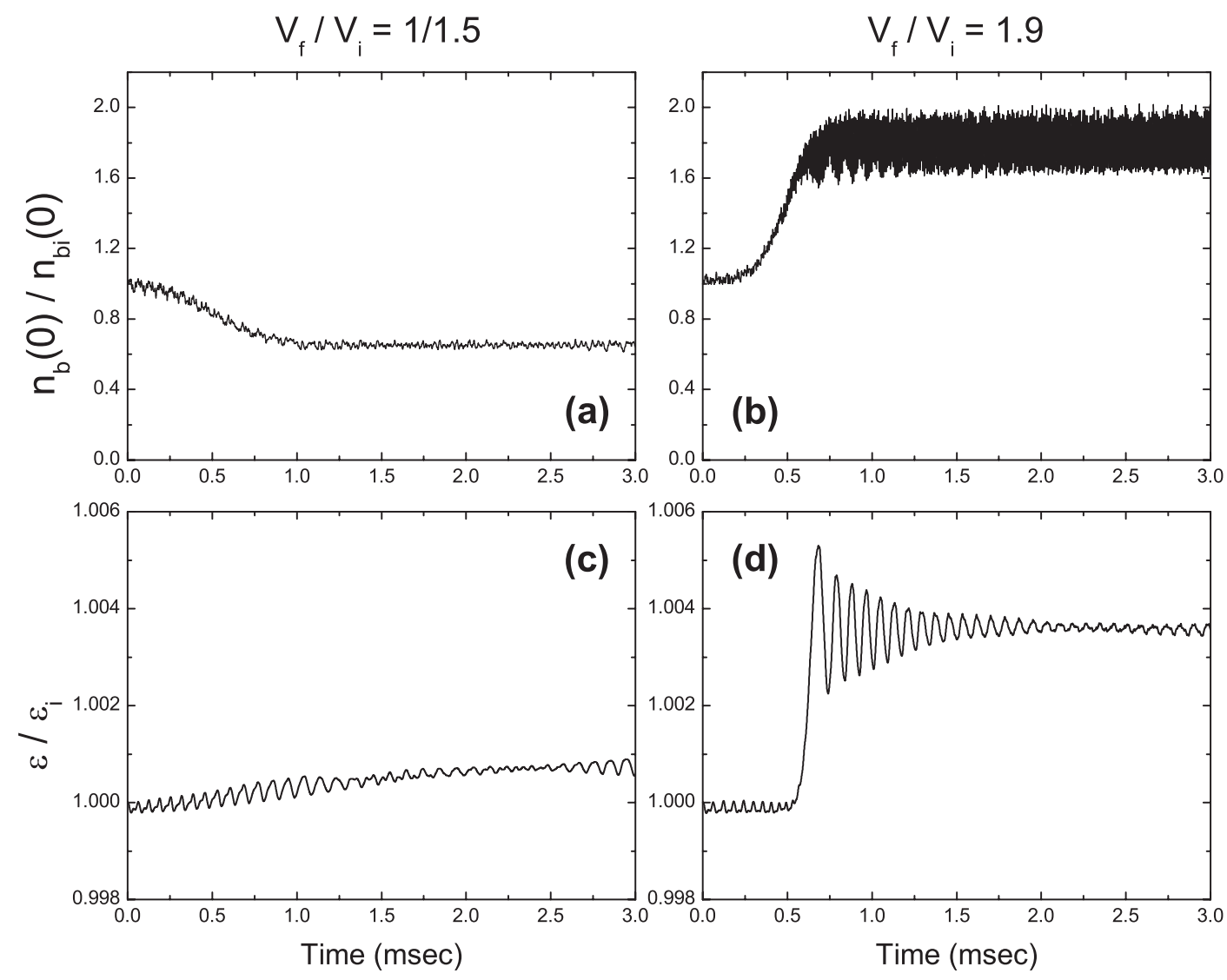

FIG. 12. 2D WARP simulations for an alternating-gradient quadrupole lattice. The evolution of the normalized on-axis density $n_{b}(0) / n_{b i}(0)$ and normalized transverse emittance $\epsilon / \epsilon_{i}$ are shown during an adiabatic transition process with $\sigma_{v i}^{s f}=49.8^{\circ}$ and $f=$ $60 \mathrm{kHz}$. Frames (a) and (c) correspond to the case with $V_{f} / V_{i}=1 / 1.5$ and $N_{t}=40$, and frames (b) and (d) correspond to the case with $V_{f} / V_{i}=1.9$ and $N_{t}=20$.

\section{SUMMARY AND CONCLUSIONS}

Experimental studies of the transverse compression of a long charge bunch have been performed in the Paul trap simulator experiment (PTSX). By applying voltage waveform amplitude changes, the non-neutral ion plasma trapped in the PTSX device is compressed (or expanded) following the same transverse dynamics as an intense beam pulse propagating through an AG focusing system. Modest changes $(\$ 20 \%)$ in voltage amplitude compress or expand the beam slightly. In this case, the difference is small between making the transition instantaneously or adiabatically. Most interestingly, when the voltage waveform amplitude is increased by more than $20 \%$, adiabatic compression of the waveform over only about four lattice periods is adequate to assure that the charge bunch remains well-matched after the compression. The experimental data for adiabatic compression are consistent with analytical estimates and 2D WARP simulations, showing that the emittance remains approximately constant during the transition. On the other hand, an instantaneous transition induces beam mismatch, which is accompanied by emittance growth.
Future experiments with other choices of waveform (e.g., a focus-drift-defocus-drift, or FODO, lattice) for the focusing field of an AG transport system are expected to further improve our basic understanding of transverse beam compression for high-intensity accelerator applications.

\section{ACKNOWLEDGMENTS}

This research was supported by the U.S. Department of Energy. The authors would like to thank Mr. A. Carpe for his excellent technical support, and Dr. D. P. Grote and Dr. E. A. Startsev for useful discussions regarding the WARP simulations.

[1] R.C. Davidson and H. Qin, Physics of Intense Charged Particle Beams in High Energy Accelerators (World Scientific, Singapore, 2001).

[2] M. Reiser, Theory and Design of Charged Particle Beams (Wiley, New York, 1994).

[3] T. Wangler, RF Linear Accelerators (Wiley, New York, 1998). 
[4] A. W. Chao, Physics of Collective Beam Instabilities in High Energy Accelerators (Wiley, New York, 1993).

[5] M. Dorf, R. C. Davidson, and E. A. Startsev, Phys. Rev. ST Accel. Beams 9, 034202 (2006).

[6] A. B. Sefkow and R. C. Davidson, Phys. Rev. ST Accel. Beams 9, 090101 (2006).

[7] T.P. Wangler, K. R. Crandall, R. Ryne, and T.S. Wang, Phys. Rev. ST Accel. Beams 1, 084201 (1998).

[8] C. K. Allen et al., Phys. Rev. Lett. 89, 214802 (2002).

[9] E. Henestroza et al., Phys. Rev. ST Accel. Beams 7, 083501 (2004).

[10] W. Paul and H. Steinwedel, Z. Naturforsch. A 8, 448 (1953).

[11] R. C. Davidson, H. Qin, and G. Shvets, Phys. Plasmas 7, 1020 (2000).

[12] E.P. Gilson, R.C. Davidson, P.C. Efthimion, and R.
Majeski, Phys. Rev. Lett. 92, 155002 (2004).

[13] E. P. Gilson, M. Chung, R. C. Davidson, P. C. Efthimion, R. Majeski, and E. A. Startsev, Nucl. Instrum. Methods Phys. Res., Sect. A 544, 171 (2005).

[14] M. Reiser, J. Appl. Phys. 70, 1919 (1991).

[15] M. Chung, E. P. Gilson, M. Dorf, R. C. Davidson, P. C. Efthimion, and R. Majeski, Phys. Rev. ST Accel. Beams 10, 014202 (2007).

[16] S. M. Lund and S. R. Chawla, Nucl. Instrum. Methods Phys. Res., Sect. A 561, 203 (2006).

[17] A. Friedman, D. P. Grote, and I. Haber, Phys. Fluids B 4, 2203 (1992).

[18] E. P. Gilson, M. Chung, R. C. Davidson, M. Dorf, P. C. Efthimion, D. P. Grote, R. Majeski, and E. A. Startsev, Nucl. Instrum. Methods Phys. Res., Sect. A 577, 117 (2007). 